\title{
Application of Modified Finite-Difference Formulas to the Analysis of $z$-Variant Rib Waveguides
}

\author{
Junji Yamauchi, Member, IEEE, Shu Nakamura, and Hisamatsu Nakano, Fellow, IEEE
}

\begin{abstract}
Modified finite-difference formulas for a general position of an interface are applied to the propagating beam analysis of $z$-variant rib waveguides. The modified formula based on the semivectorial $\boldsymbol{H}$-field is found to be more insensitive to variation in an interface position than that on the $E$-field. A discretization error is satisfactorily reduced in tilted and tapered rib waveguides.
\end{abstract}

Index Terms-Finite-difference methods, optical beam propagation, optical waveguides.

\section{INTRODUCTION}

I $\mathrm{T}$ IS imperative to reduce the discretization error in the propagating beam analysis of a $z$-variant step-index optical waveguide. There are some techniques to tackle this problem. One of them is to use a modified grid that is adaptive to a waveguide geometry. Another is to use a finite-difference (FD) formula that allows a general position of an interface.

In the former technique, an oblique [1], a more advanced bi-oblique [2], and a tapered [3] coordinate have been developed and tested in the finite-difference beam-propagation method (BPM). A tapered-grid approach based on cylindrical coordinates has been used in the BPM based on the method of lines [4]. These modified grid techniques can successfully reduce the discretization error, although the connection between different coordinates is often required depending on the model to be analyzed.

On the other hand, the latter technique has the advantage that Cartesian coordinates are uniformly used, so that an inherent error caused by a nonuniform grid is completely eliminated. Nevertheless, no attempt has been made for the analysis of a three-dimensional (3-D) $z$-variant step-index waveguide except for our preliminary report [5]. This is due to the fact that the rigorous derivation of an FD formula is difficult for 3-D step-index waveguides.

In this letter, we demonstrate that a simple modified FD formula developed for the semivectorial $\mathrm{H}$-field [6] can successfully be employed for the propagating beam analysis of $z$-variant rib waveguides with a reduced discretization error. After a brief explanation of the modified FD formula, we discuss the difference between the results obtained from the Eand $H$-field formulas and clarify the advantage of the $H$-field formula through the eigenmode analysis. In the propagating beam analysis, tilted and tapered rib waveguides are treated.

Manuscript received February 11, 2000.

The authors are with the College of Engineering, Hosei University, Koganei, Tokyo 184, Japan.

Publisher Item Identifier S 1041-1135(00)06279-0.

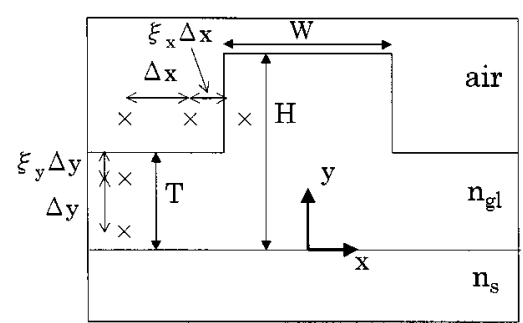

Fig. 1. Rib waveguide geometry: $W=3.0 \mu \mathrm{m}, H=1.0 \mu \mathrm{m}, T=0.5 \mu \mathrm{m}$, $n_{g l}=3.44, n_{s}=3.40$

The field profile and the guided-mode power at the output are compared with those obtained using a staircase approximation.

\section{PROPERTIES OF MODIFIED FD Formulas}

Modified FD formulas, which are regarded as the extension of Stern's formula [7], have been developed for semivectorial fields by several authors [6], [8]-[11]. The formulas are derived assuming a planar geometry where all the discontinuities are either parallel or orthogonal to axes. This assumption is, therefore, approximate when analyzing a 3-D waveguide. We should note, however, that the paraxial wave in a slowly $z$-variant waveguide is usually treated in the propagating beam analysis; the interface is nearly parallel or orthogonal to axes. Furthermore, the modified formula allows us to use an arbitrary interface position. These facts encourage us to apply the modified formulas to the propagating beam analysis of a $z$-variant step-index waveguide.

We consider the rib waveguide shown in Fig. 1, which has been used as a classical benchmark [12]. The configuration parameters are $n_{s}=3.40, n_{g l}=3.44$, rib width $W=3.0 \mu \mathrm{m}$, central rib height $H=1.0 \mu \mathrm{m}$, and lateral height $T=0.5 \mu \mathrm{m}$. Suppose that a discontinuity is located between points $i$ and $i+1$ and the interface is at distance $\xi_{x} \Delta x$ (with $0<\xi_{x}<1$ ) from point $i$. Using Taylor-series expansions of the $E$ - or $H$-field near the discontinuity and the boundary conditions at the interface, we have derived the modified FD formulas that allow a general position of an interface. For instance, the FD formula based on the $H$-field [6] is expressed as

$$
\frac{\partial^{2} \phi_{i}}{\partial x^{2}}=\frac{a \phi_{i-1}+b \phi_{i}+c \phi_{i+1}}{d \Delta x^{2}}+e \frac{\Delta x}{3} \frac{\partial^{3} \phi_{i}}{\partial x^{3}}+O\left(\Delta x^{2}\right)
$$

where $a=1+(\theta-1)\left(1-\xi_{x}\right)+m \Delta x^{2} \Gamma ; b=-2-(\theta-1)(1-$ $\left.\xi_{x}\right)-m \Delta x^{2}(1+\Gamma) ; c=1 ; d=1+0.5(\theta-1)\left(1-\xi_{x}\right)\left(2 \xi_{x}+\right.$ 1) $+0.5 m \Delta x^{2} \Gamma$; and $e=-(\theta-1) \xi_{x}\left(1-\xi_{x}\right)\left(2 \xi_{x}-1\right) / d$, in which $m$ and $\Gamma$ are defined in [6]. The coefficient $\theta=n_{i+1}^{2} / n_{i}^{2}$ must be unity when the normal component is treated. Equation (1) is also used for the second derivative with respect to $y$. A formula similar to (1) has also been derived for the $E$-field [8]. It 


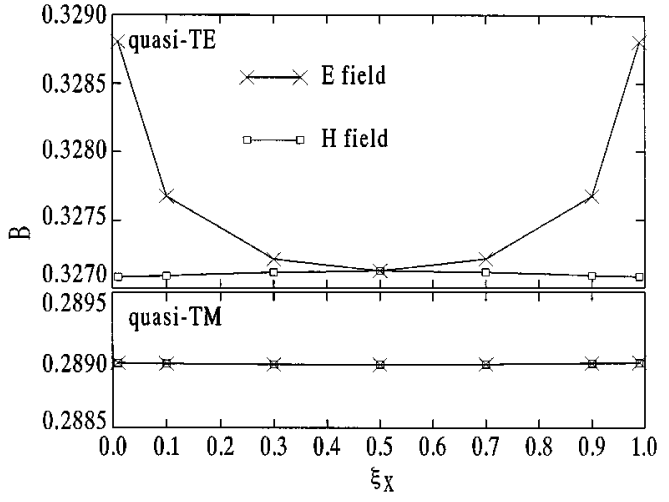

Fig. 2. Normalized propagation constant $B$ as a function of $\xi_{x}\left(\xi_{y}=0.5\right)$.

should be noted, however, that the formula based on the $H$-field has some advantages, as will be seen in Figs. 2 and 3.

The first-order term in (1) is neglected in this analysis. Since the coefficient $e$ vanishes for $\theta=1,(1)$ is second-order accurate as long as the normal component is treated. Although the present analysis does not ensure second-order accuracy for the tangential component $(\theta \neq 1)$, the first-order term has a much smaller effect than the error caused by a staircase approximation [13]. This leads to a significant improvement in accuracy even for $\theta \neq 1$.

To assess how the results obtained from the modified formulas for the $E$ - and $H$-fields depend on the interface position, we first make the eigenmode analysis for both the quasi-TE and quasi-TM modes at a wavelength of $\lambda=1.15 \mu \mathrm{m}$. The transverse sampling width is fixed to be $\Delta(=\Delta x=\Delta y)=$ $0.025 \mu \mathrm{m}$, and the total number of sampling points is taken to be $N_{x} \times N_{y}=320 \times 180$. The modified formulas are applied to the alternating-direction implicit BPM with the imaginary-distance procedure using a unit amplitude as a starting field [6].

Fig. 2 shows the normalized propagation constant $B=\left(n_{\text {eff }}^{2}-n_{s}^{2}\right) /\left(n_{g l}^{2}-n_{s}^{2}\right)$ as a function of $\xi_{x}$. It is clear that the $E$-field formula gives the same results as those obtained from the $H$-field formula in the quasi-TM mode. It should be noted, however, that the results from the $E$-field are sensitive to the position of the interface in the quasi-TE mode, while the results from the $H$-field are insensitive to the position. The dependency of the $E$-field results on the interface position is probably due to the fact that the $E$-field becomes singular at the lower corners. This dependency has first been found by Vassallo [9]. To eliminate this dependency, he developed a six-point formula for the $E$-field Laplacian. It should be noted that the application of the six-point formula to the BPM requires an extra computational effort. It is worth mentioning that the dependency on the interface position can almost be eliminated by the use of the simple FD formula based on the $H$-field.

Similar behavior observed in Fig. 2 is found in Fig. 3, in which $B$ is expressed against $\xi_{y}$ with $\xi_{x}=0.5$. We again find that the data obtained for the $H$-field are more stable than those for the $E$-field. Note that asymmetry of the waveguide geometry with respect to the $x$ axis causes slight variation of $B$ for the quasi-TE mode.

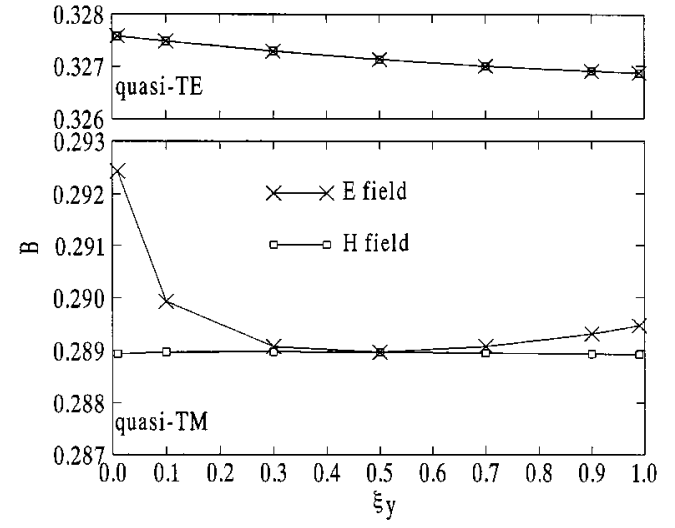

Fig. 3. Normalized propagation constant $B$ as a function of $\xi_{y}\left(\xi_{x}=0.5\right)$.

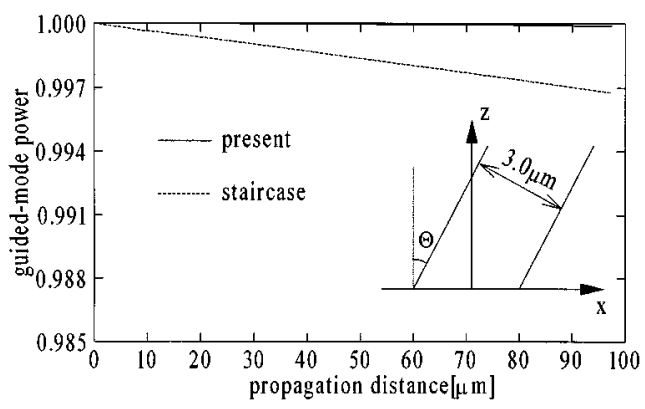

Fig. 4. Fundamental-mode power as a function of propagation distance.

\section{Propagating Beam Analysis}

Since the superiority of the $H$-field formula is demonstrated, the $H$-field formula is applied to the propagating beam analyses of $z$-variant structures. We first study the propagating beam in a tilted rib waveguide. The waveguide whose configuration parameters are identical to those in Fig. 1 is tilted by angle $\Theta$. Since the configuration changes only in the $x-z$ plane, we fix $\xi_{y}$ to be 0.5 , while $\xi_{x}$ is variable. Fig. 4 shows the guided-mode power as a function of propagation distance. The quasi-TE mode is tested, and the tilt angle is typically chosen to be $\Theta=1^{\circ}$. The computational parameters are chosen to be $\Delta=0.05 \mu \mathrm{m}$ and $\Delta z \simeq$ $0.072 \mu \mathrm{m}$. Ideally, the guided-mode power should be unity regardless of the propagation distance. It is clearly shown that the present scheme maintains the initial guided-mode power. In contrast, the use of the staircase approximation $\left(\xi_{x}\right.$ is fixed to be 0.5 ) results in gradual deterioration with an increase in the propagation distance.

We next consider a tapered rib waveguide. The rib width is tapered from 3 to $2 \mu \mathrm{m}$, as shown in the inset of Fig. 5. Calculation is made for $\Delta z=0.05 \mu \mathrm{m}$. Other configuration and computational parameters are the same as those used in Fig. 4. Fig. 5 shows the guided-mode power at the output as a function of $\Delta$. Note that the guided-mode power dose not become unity because of radiation loss along the tapered waveguide. For comparison, the data obtained from the staircase approximation are also shown. The present technique shows rapid convergence behavior.

Fig. 6 illustrates the output field profiles for $\Delta=0.1 \mu \mathrm{m}$. Appreciable numerical noise is found for the staircase approxi- 


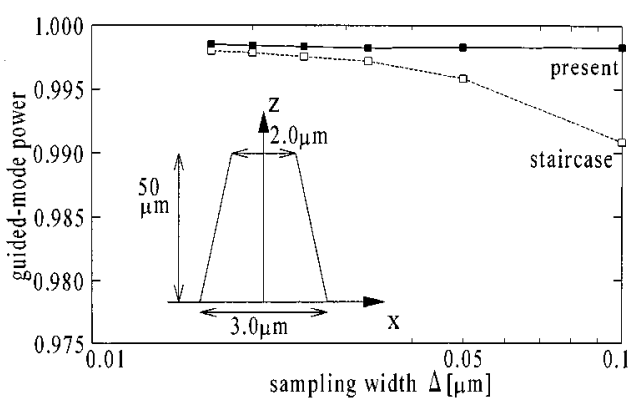

Fig. 5. Convergence behavior of the fundamental-mode power.
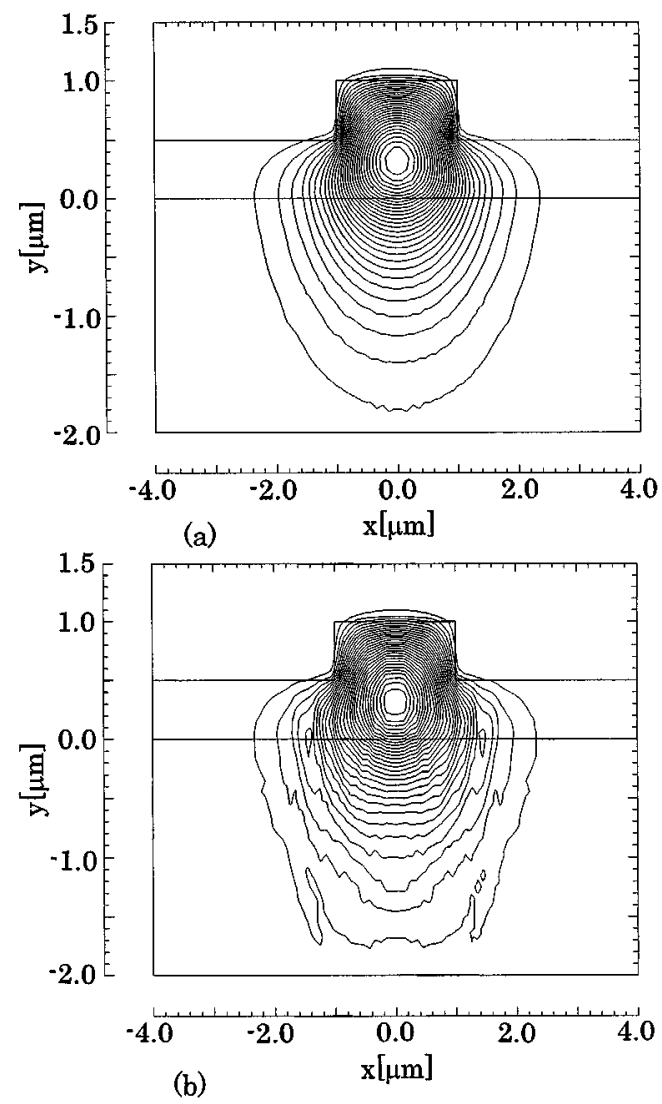

Fig. 6. Field profiles observed at the output of the tapered rib-waveguide: (a) present $\left(\xi_{y}=0.5\right)$ and (b) staircase approximation $\left(\xi_{x}=\xi_{y}=0.5\right)$.

mation. The use of the present formula greatly contributes to a reduction in the numerical noise.

\section{CONCLUSIONS}

The simple modified finite-difference formula that allows a general position of an interface has been applied to the propagating beam analysis of rib waveguides with particular emphasis on reducing the discretization error. After confirming the fact that the results obtained from the $H$-field formula are almost independent of the interface position, we calculate the propagating beams in tilted and tapered waveguides. Numerical results clearly show a reduction in the numerical noise of the field profile with fast convergence as a function of transverse sampling width.

\section{REFERENCES}

[1] J. Yamauchi, J. Shibayama, and H. Nakano, "Propagating beam analysis based on the implicit finite-difference method using oblique coordinate system," in OSA Integr. Photon. Res. Tech. Dig., 1994, pp. 19-21.

[2] P. Sewell, T. M. Benson, T. Anada, and P. C. Kendall, "Bi-oblique propagation analysis of symmetric and asymmetric Y-junctions," J. Lightwave Technol., vol. 15, no. 4, pp. 688-696, 1997.

[3] S. Sujecki, P. Sewell, T. M. Benson, and P. C. Kendall, "Novel beam propagation algorithms for tapered optical structures," J. Lightwave Technol., vol. 17, no. 11, pp. 2379-2388, 1999.

[4] S. Helfert and R. Pregla, "New developments of a beam propagation algorithm based on the method of line," Opt. Quantum Electron., vol. 27, pp. 943-950, 1995.

[5] J. Yamauchi, S. Nakamura, and H. Nakano, "Propagating beam analysis based on the finite-difference formula for a general position of interfaces," in OSA Integr. Photon. Res. Dig., 1999, pp. 101-103.

[6] J. Yamauchi, G. Takahashi, and H. Nakano, "Modified finite-difference formula for semivectorial $\mathrm{H}$-field solutions of optical waveguide," IEEE Photon. Technol. Lett., vol. 10, pp. 1127-1129, Aug. 1998.

[7] M. S. Stern, "Semivectorial polarized H field solutions for dielectric waveguides with arbitrary index profiles," Proc. Inst. Elect. Eng. J., vol. 135, no. 5, pp. 333-338, 1988.

[8] J. Yamauchi, M. Sekiguchi, O. Uchiyama, J. Shibayama, and H. Nakano, "Modified finite-difference formula for the analysis of semivectorial modes in step-index optical waveguides," IEEE Photon. Technol. Lett., vol. 9, pp. 961-963, July 1997.

[9] C. Vassallo, "Improved finite difference derivation of semi-vectorial modes in 3-D step-index waveguides," in Proc. ECOC'92, 1992, pp. $565-568$.

[10] — - "Improvement of finite difference methods for step-index optical waveguides," Proc. Inst. Elect. Eng. J., vol. 139, no. 2, pp. 137-142, 1992.

[11] R. Stoffer and H. J. W. M. Hoekstra, "Efficient interface conditions based on a 5-point finite difference operator," Opt. Quantum Electron., vol. 30, no. 5/6, pp. 375-383, 1998.

[12] C. Vassallo, "1993-1995 optical mode solvers," Opt. Quantum Electron., vol. 29, no. 2, pp. 95-114, 1997.

[13] J. Yamauchi, K. Matsubara, T. Tsuda, and H. Nakano, "Norm-conserving finite-difference beam propagation method for TM wave analysis in step-index waveguides," J. Lightwave Technol., vol. 18, no. 5, pp. 721-728, 2000. 